

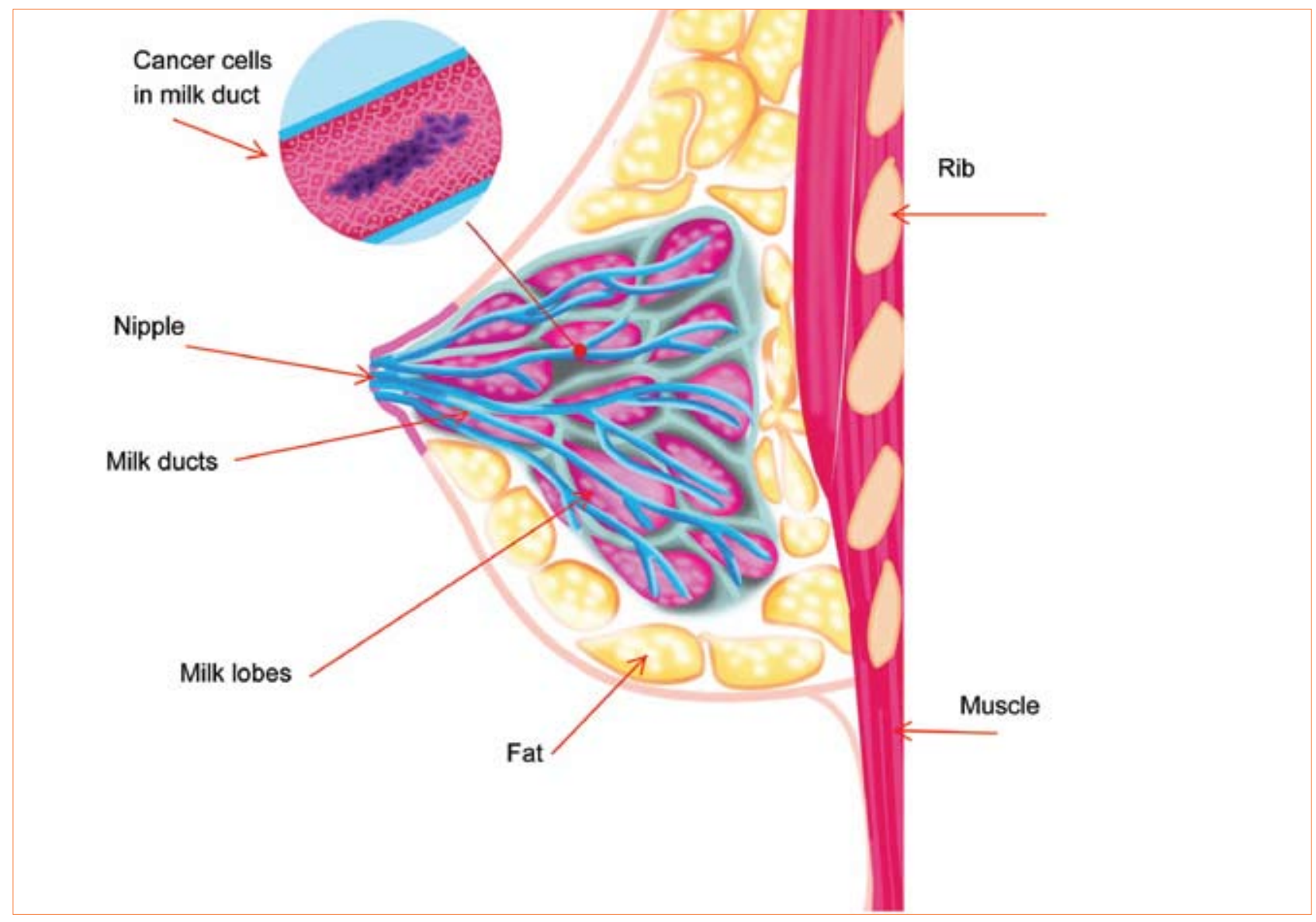

\section{What are the symptoms of DCIS?}

Most people with DCIS have no symptoms at all. You may have a lump, a thickening of tissue, or a discharge from the nipple. Rarely, you may get a type of rash involving the nipple. This is known as Paget's disease of the breast.

\section{How is DCIS diagnosed?}

As DCIS does not usually have any symptoms, most cases are diagnosed from a mammogram (breast X-ray). The mammogram shows a cluster of small white dots. These white dots contain calcium and are known as microcalcifications. Not all microcalcifications turn out to be DCIS. To confirm a diagnosis, a biopsy (removal of a piece of breast tissue) will be taken using the mammogram to guide the radiologist to the area of microcalcification. This is called a stereotactic core biopsy.
If you have symptoms such as a lump or nipple discharge, you will have a range of tests. These may include a mammogram, ultrasound and core biopsy to check for abnormal cells.

\section{Are there different types of DCIS?}

There are a number of different types of DCIS. They can be divided into high, intermediate, and low-grade DCIS. This grading is based on what the cells look like under the microscope. If DCIS is left untreated, the cells may spread from the ducts into the surrounding breast tissue and become an invasive cancer. This means that they can spread to other parts of your body. It is thought that low-grade DCIS is less likely to become an invasive cancer than high-grade DCIS.

Having a diagnosis of DCIS means you have a slightly higher risk of getting cancer elsewhere in the same breast or in your other breast. As a result, you will have regular screening mammograms after your treatment. 


\section{What is the cause of DCIS?}

The exact cause of DCIS is unknown, but there are certain risk factors that may increase your chance of getting it. Even so, women with no risk factors can develop DCIS, while women with risk factors might not. More patients are being diagnosed with DCIS in Ireland since breast screening became available. Now all women from the ages of 50 to 64 are routinely offered a free mammogram every 2 years. Finding very early disease like DCIS is one of the main aims of the screening programme.

Possible risk factors for DCIS include:

- Getting older: Your risk increases with age.

- Hormone factors: Your risk is higher if your period started early, if your menopause started late, or if you have had no children or had them later in life.

- Some breast conditions: Your risk is higher if you had breast cancer yourself in the past or a benign condition called ADH (atypical ductal hyperplasia).

- Family history of breast cancer: In a small number of people, there is an abnormal gene that can cause breast cancer.

- Lifestyle factors: Drinking alcohol more than the recommended daily limit for women ( 2 units) and being overweight after the menopause might increase your risk.

\section{What treatment will I be offered?}

The aim of treatment is to remove the early cancer cells and stop them from growing into invasive cancer. The treatment offered to you will depend on factors such as the extent of DCIS and the grade. Usually, treatment involves surgery and sometimes other follow-on treatments.

\section{Surgery}

As for most types of breast cancer you will be offered surgery as your first treatment.

You will be advised by your surgeon on the

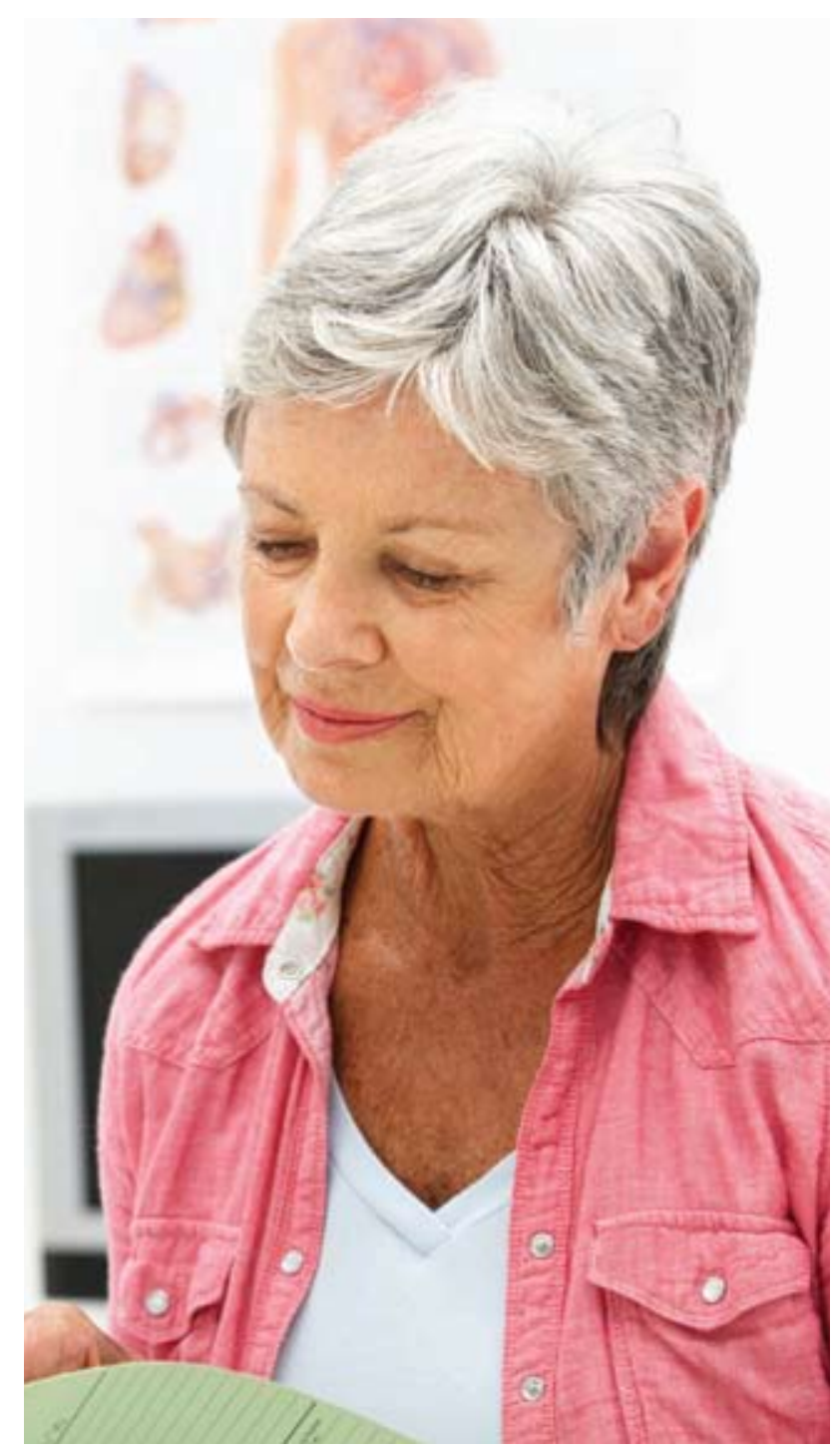

best option for you after your results have been discussed by a team of doctors, nurses and other health professionals. The type of surgery could range from wide local excision to mastectomy with or without breast reconstruction. Wide local excision means a lumpectomy, which involves removing the area of DCIS and an area of normal tissue around it. A mastectomy means your whole breast is removed. If you are offered breast reconstruction, National Cancer Helpline on 1800 200700 and speak to a specialist nurse or ask for a copy of the breast reconstruction booklet.

If the pathologist (the doctor who examines the tissue sample in the laboratory) finds the cancer has spread outside the duct, this will affect the treatment you are offered. 


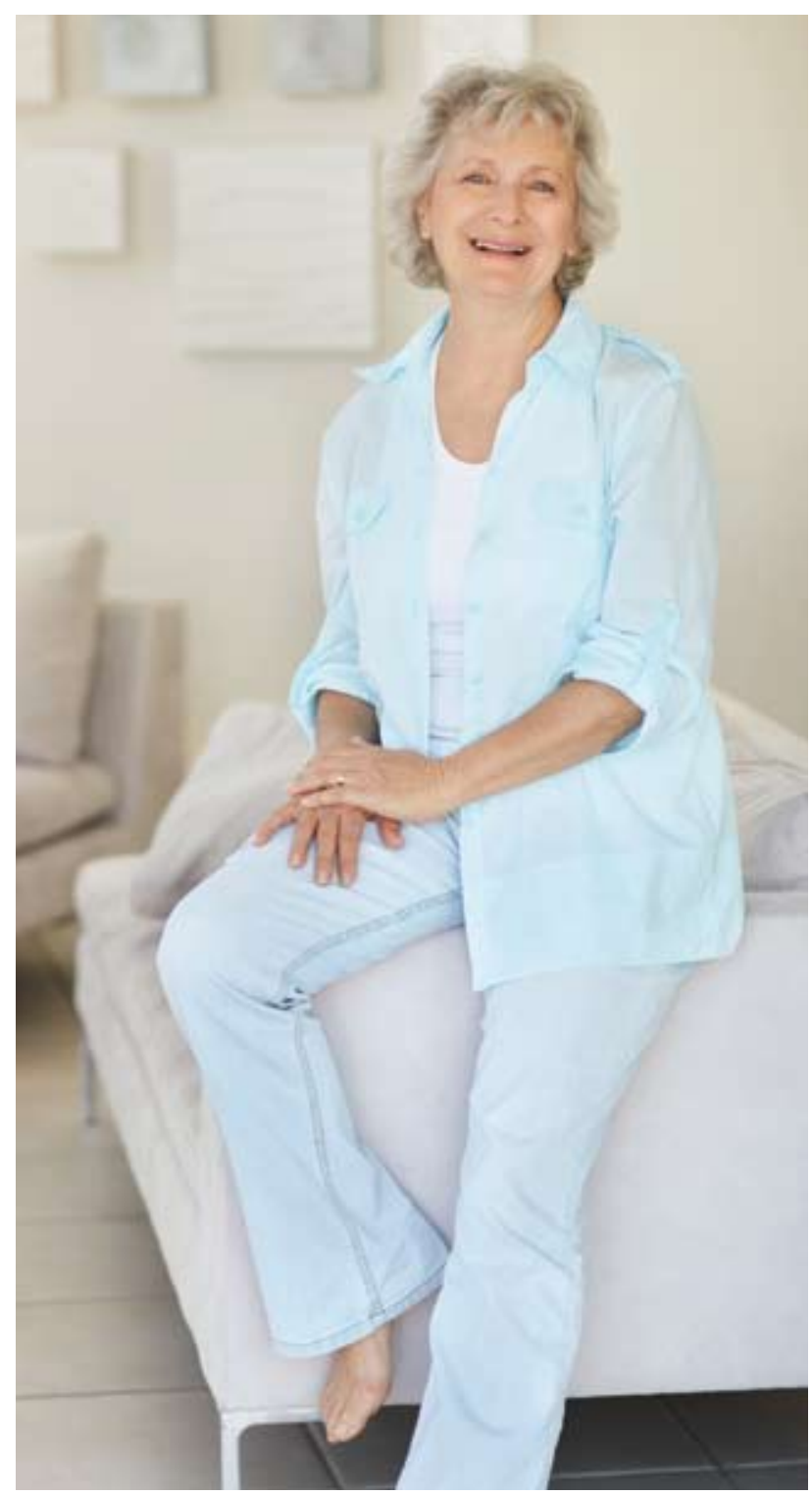

\section{Wire-guided localisation biopsy}

For DCIS that cannot be felt, wire-guided localisation is done on the morning of surgery. This helps your surgeon pinpoint the exact area to be removed during surgery. The radiologist (specialist in reading $\mathrm{X}$-rays and scans) uses a mammogram or ultrasound as a guide to put a needle into the area of microcalcification in your breast. This can take up to an hour and can be a little uncomfortable. Once the wire is in place, it is covered with a comfortable padded dressing and left there until surgery.

\section{Sentinel lymph node biopsy}

It is unusual to find any cancer cells in the lymph nodes with a diagnosis of DCIS. But in some cases a sentinel lymph node biopsy may be advised, especially if the area of DCIS is large and high grade. This is a way of checking the lymph nodes in your armpit for cancer cells. The sentinel node is the first one to drain lymph fluid from your breast, so it is the most likely to contain any cancer cells. This test is usually carried out at the same time as breast surgery.

Usually on the morning of surgery, you will be brought to the X-ray department to have a small amount of harmless radioactive liquid injected into your breast, usually around the nipple. A scan usually follows some time later and shows the lymph nodes up on a screen.

Then your skin can be marked to show where the nodes are.

During the operation a second method using a blue dye can be injected into the area.

This is an extra way to help show where the nodes are. Your surgeon removes them and they are sent to the laboratory. These nodes are examined by the pathologist to make sure there are no cancer cells present. If the sentinel node contains cancer cells, it means there is an invasive cancer in your breast.

\section{Other treatments or adjuvant treatments}

Adjuvant treatments are treatments given as well as surgery to reduce the risk of DCIS coming back or an invasive cancer developing. These include radiotherapy and hormone therapy. Chemotherapy is not used for DCIS. A lumpectomy is likely to be followed up with radiotherapy unless the area of DCIS was very small and/or low grade. Call the National Cancer Helpline on Freefone 1800200700 and ask for our factsheet on radiotherapy.

If the type of DCIS you have is oestrogen receptor positive (grows with the help of the female hormone oestrogen), you may be offered hormone therapy. This is sometimes offered as part of a clinical trial. This will also depend on other factors such as the grade of the DCIS. For more information call the National Cancer Helpline on Freefone 1800200700. 


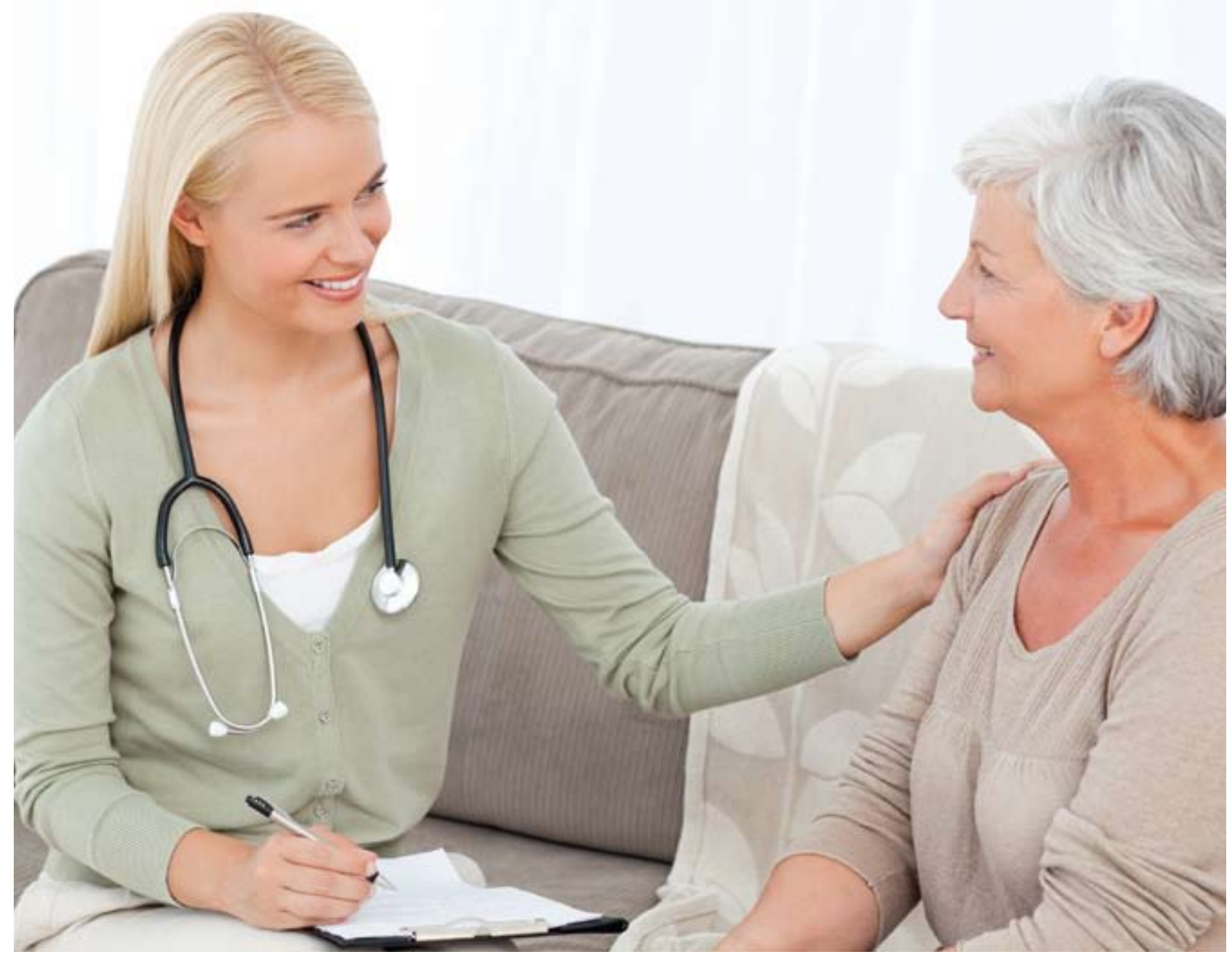

\section{Coping with breast cancer}

Finding out that you have breast cancer can leave you feeling a range of emotions. For example, fear, shock, sadness and anger are all common feelings at this time.

Although DCIS is a very early and treatable form of breast cancer, you may still feel anxious and rather negative. Remember that there are people who can support you so do not be afraid to ask for help.

Do let other people know how you are feeling, particularly your family and friends, so that they can be more supportive. It can also help to discuss your feelings or worries with your breast care nurse or specialist.

\section{Further Support}

You might find it easier to share your feelings with someone who has had a similar experience to you. For example, Reach to Recovery is a programme set up to help and support women who have recently had a breast cancer diagnosis. The programme involves personal contact between you and a Reach to Recovery volunteer - a woman who has had treatment for breast cancer. Carefully selected and fully trained volunteers are available to provide advice and reassurance at a time when you are most in need of both. For more information on individual support or support groups in your area, call the National Cancer Helpline on 1800200700. 


\section{CANCER INFORMATION FACTSHEET}

\section{Useful Organisations}

\section{American Cancer Society}

Website: www.cancer.org

\section{Irish Nutrition \& Dietetic Institute}

Website: www.indi.ie

\section{Irish Osteoporosis Society}

Website: www.osteoporosis.ie

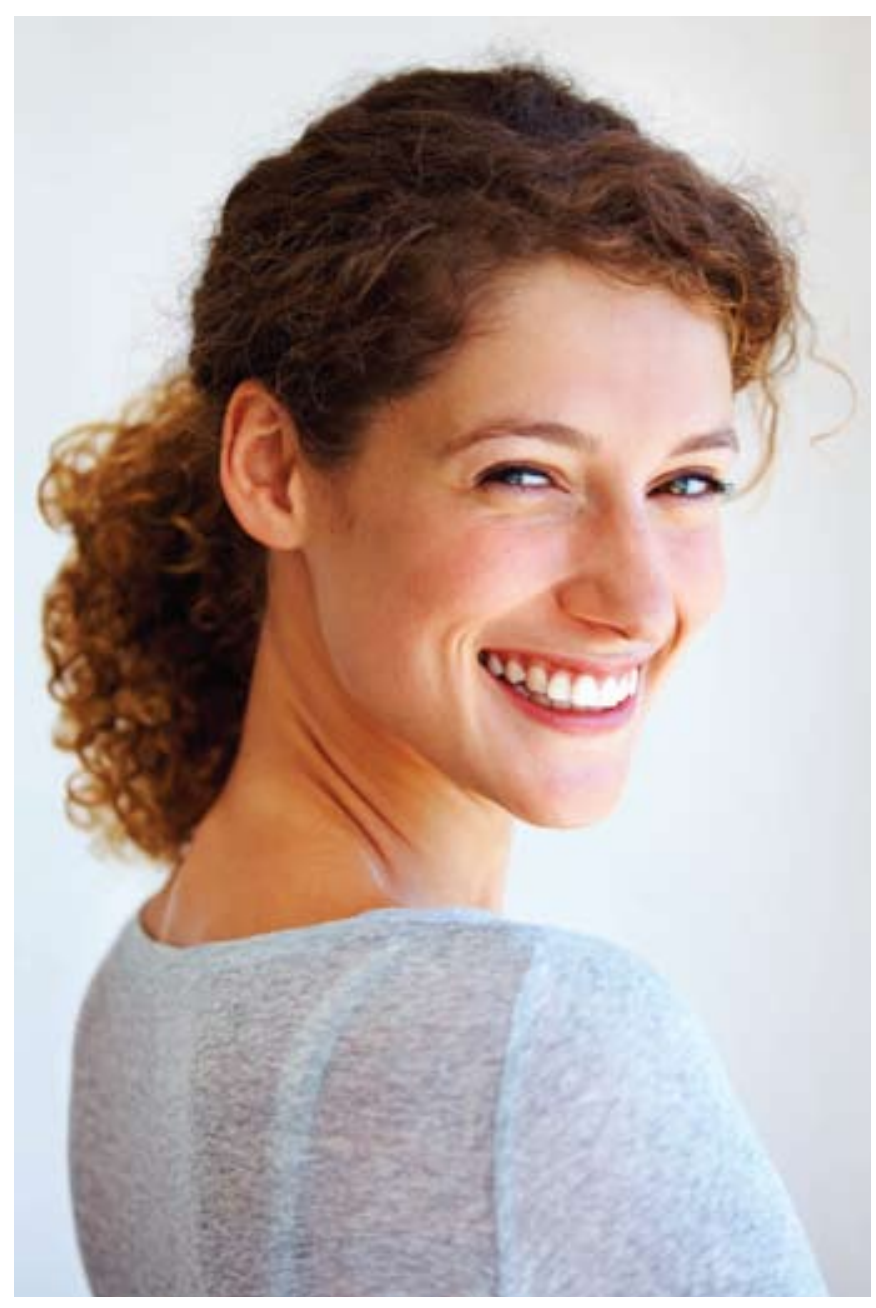

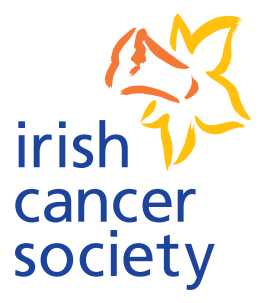

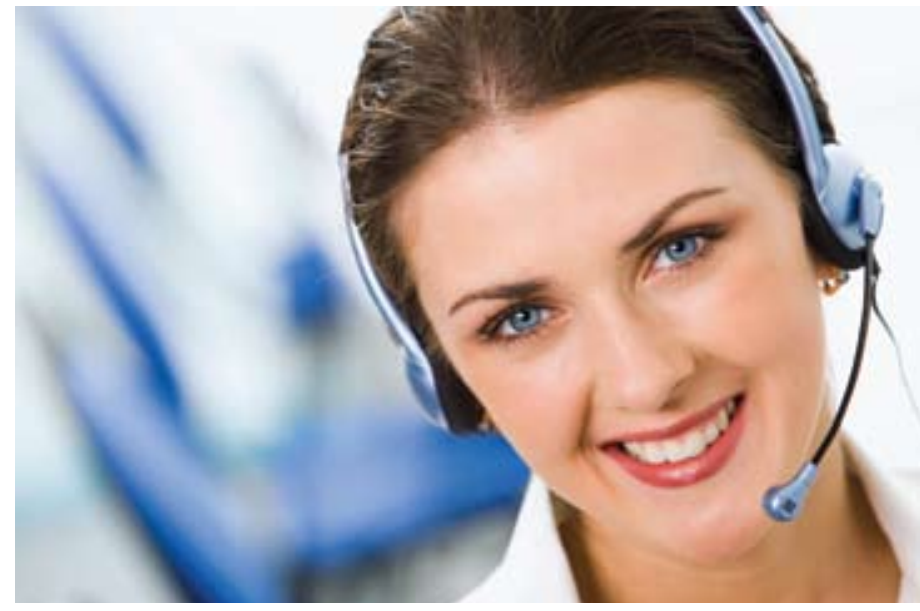

\section{FURTHER INFORMATION}

For more information on DCIS or for confidential advice from our cancer nurse specialists, call the National Cancer Helpline Freefone

\section{0}

(Monday-Thursday, 9am-7pm; Friday, 9am-5pm) or email helpline@irishcancer.ie

\section{Irish Cancer Society}

43/45 Northumberland Road, Dublin 4

Tel: (01) 2310500

Fax: (01) 2310555

Email: helpline@irishcancer.ie

Website: www.cancer.ie

Published by the Irish Cancer Society.

(C) Irish Cancer Society, 2007, revised 2011

Next revision: 2013

All rights reserved. No part of this publication may be reproduced or transmitted, in any form or by any means, electronic or mechanical, including photocopying, recording or any other information storage and retrieval system, without permission in writing from the Irish Cancer Society. 